\title{
Prognostic Factors in Geriatric Patients with Metastatic Colon Cancer
}

\section{Kenji Ina ${ }^{1 *}$, Megumi Kabeya², Shu Yuasa ${ }^{2}$, Yuko Kato ${ }^{3}$, Satoshi Kayukawa4, Takae Kataoka4, Ryuichi Furuta ${ }^{1}$}

\author{
${ }^{1}$ Department of Medical Oncology, Nagoya Memorial Hospital, Nagoya, Japan \\ ${ }^{2}$ Department of Phamacy, Nagoya Memorial Hospital, Nagoya, Japan \\ ${ }^{3}$ Medical Social Work Consultation Room, Nagoya Memorial Hospital, Nagoya, Japan \\ ${ }^{4}$ Department of Clinical Oncology, Nagoya Memorial Hospital, Nagoya, Japan \\ Email: *kina@hospy.or.jp
}

How to cite this paper: Ina, K., Kabeya, M., Yuasa, S., Kato, Y., Kayukawa, S., Kataoka, T. and Furuta, R. (2019) Paper Title. Journal of Cancer Therapy, 10, 779-788. https://doi.org/10.4236/jct.2019.1010065

Received: July 29, 2019

Accepted: September 23, 2019

Published: September 26, 2019

Copyright (c) 2019 by author(s) and Scientific Research Publishing Inc. This work is licensed under the Creative Commons Attribution International License (CC BY 4.0).

http://creativecommons.org/licenses/by/4.0/

\begin{abstract}
Backgrounds: Colorectal cancer is an important contributor to cancer morbidity and mortality. Given that many older adult patients often have concomitant diseases and impairments in organ function, they are at increased risk for chemotherapy toxicity. Therefore, it is important to identify prognostic factors in older patients undergoing chemotherapy. Methods: We performed a retrospective chart review of colorectal cancer patients treated at Nagoya Memorial Hospital between 2012 and 2017 and selected those who received chemotherapy for metastatic colon cancer. Overall survival was calculated from the beginning of chemotherapy until death or the most recent follow-up date. We used the Kaplan-Meier method to plot survival curves and performed a statistical comparison using a log-rank test. In addition, multivariate analysis was performed using stepwise Cox proportional hazards models. Finally, a comprehensive geriatric assessment was conducted for older patients. The chart review was approved by the ethics committee of Nagoya Memorial Hospital. Results: The overall survival of metastatic colon cancer patients was not markedly different between patients aged $<65$ years $(\mathrm{N}=17)$ and those aged $\geq 65$ years of age $(\mathrm{N}=37)$. Neither lesion site nor the $R A S$ status affected survival, whereas the usage of three kinds of cytotoxic agents prolonged longevity. In a multivariate analysis of patients $\geq 65$ years of age, the only independent prognostic factor for survival was the functional capacity, as measured by the Tokyo Metropolitan Institute of Gerontology (TMIG) index of competence. Conclusions: A combination of the comprehensive geriatric assessment and TMIG index was useful for predicting the longevity in patients with metastatic colon cancer $\geq$ 65 years of age.
\end{abstract}




\section{Keywords}

Metastatic Colon Cancer, Chemotherapy, Comprehensive Geriatric Assessment, TMIG Index of Competence

\section{Introduction}

Colorectal cancer is an important threat to human health and the third most common type of cancer worldwide [1]. In the past decade, significant advances have been made in the treatment of metastatic colorectal cancer [2] [3] [4] [5]. The development of cytotoxic therapies and targeted agents has improved median survival time to more than 30 months [6]. Several prognostic factors for colorectal cancer have been examined, including performance status (PS), age, sex, and biological variables such as serum levels of lactate dehydrogenase (LDH) and serum carcinoembryonic antigen (CEA) [7] [8]. Because several critical genes and pathways play an important role in the initiation and progression of colorectal cancer [9] [10], RAS gene mutations and mismatch repair status have been associated with the prognosis of affected patients [11] [12] [13]. In addition, recent studies have suggested that the location of the primary tumor might be of prognostic value [14] [15]. Studies have shown that patients with metastatic cancer originating in the left side of the colon showed improved survival after treatment with an anti-EGFR antibody, compared to patients with cancer originating in the right side of the colon [16] [17].

In addition, cancer is associated with aging. In 2017, Japan had the world's largest aging population with an average life span of more than 80 years. Individuals aged 65 years or older account for $27.7 \%$ of the overall population. Considering that many older patients often have concomitant diseases and age-related changes in pharmacokinetics and pharmacodynamics [18], cancer chemotherapy in older patients requires particular caution [19] [20]. Although the definition of "elderly" has not been universally defined or accepted, most developed countries have the age over 65 years as the definition for an elderly person. Since it is generally recognized that chronological age is not equivalent to physiologic age, we examined the factors that are associated with overall survival (OS) in older patients (aged 65 and older) with colorectal cancer undergoing chemotherapy.

\section{Patients and Methods}

Medical records were retrospectively reviewed to examine the clinicopathological features of colorectal cancer patients (469 with colon cancer and 324 with rectal cancer) treated at Nagoya Memorial Hospital from April 2012 to March 2017. The chart review was approved by the ethics committee of the hospital. Considering the site of the primary lesion, the patients with rectal cancer were excluded to ease the interpretation of results. Accordingly metastatic colon can- 
cer patients receiving chemotherapy $(\mathrm{N}=54)$ were selected from the above 793 patients, and the following data were collected from their medical records: age, sex, PS, site of primary lesion, histological type, $R A S$ status, metastatic sites, serum CEA levels, serum LDH levels at baseline, chemotherapeutic agents administered, tumor response, and OS.

A comprehensive geriatric assessment was conducted for older patients using both an 8-item function-based scoring system (IADL) [21] and a 13-item scoring system (Tokyo Metropolitan Institute of Gerontology [TMIG] index of competence) [22]. In this study, older patients were defined as those aged 65 years and older at the time of initiation of systemic chemotherapy. Objective response to chemotherapy was evaluated using the criteria proposed by Response Evaluation Criteria in Solid Tumors [23] for metastatic lesions. The OS was calculated from the time chemotherapy was initiated to death or the most recent follow-up day. Results were compared using a log-rank test for statistical analysis. Multivariate analysis was performed using stepwise Cox proportional hazards models. The following factors were analyzed by converting the following variables into dichotomous variables: 1 ) age, $<65$ vs. $\geq 65$ years; 2) sex, male vs. female; 3) primary tumor location, left-sided vs. right-sided; 4) $R A S$ status, mutation vs. wild-type; 5) the number of cytotoxic chemotherapeutic agents, $<3$ vs. $\geq 3$; and 6) functional capacity (as measured by either IADL or TMIG index of competence), full score vs. others.

Differences with $P$-values $<0.05$ were considered statistically significant. All statistical analyses were performed using EZR (Saitama Medical Center, Jichi Medical University;

http://www.jichi.ac.jp/saitama-sct/SaitamaHP.files/statmedEN.html), which is a graphical user interface for R (The R Foundation for Statistical Computing, Vienna, Austria) [24].

\section{Results}

Our retrospective study included 54 patients with metastatic colon cancer receiving palliative chemotherapy. Figure 1 shows that OS did not differ markedly between patients aged $<65$ years $(\mathrm{N}=17)$ (median OS [days; 95\% confidence interval $\{\mathrm{CI}\}], 823$ [269 - 1467]) and $\geq 65$ years of age $(\mathrm{N}=37$, median OS, 805 [471 - 1138]). Neither RAS status nor lesion affected the survival of metastatic colon cancer patients (Figure 2, Figure 3). In contrast, the usage of three cytotoxic agents (fluoropyrimidines, oxaliplatin, and irinotecan) significantly prolonged the longevity of the affected patients (median OS [days; 95\% CI] $=1138$ [732 - 1394]), compared to those who did not receive all three drugs (median OS $=352$ [171 - 805]; $P<0.01$ ) (Figure 4). We expected that if we focused on older patients ( $\geq 65$ years of age), the number of chemotherapeutic agents and functional capacity would be associated with their OS (Figure 5 and Figure 6). However, multivariate analysis of older patients revealed that the only independent prognostic factor for survival was functional capacity, as measured by TMIG 


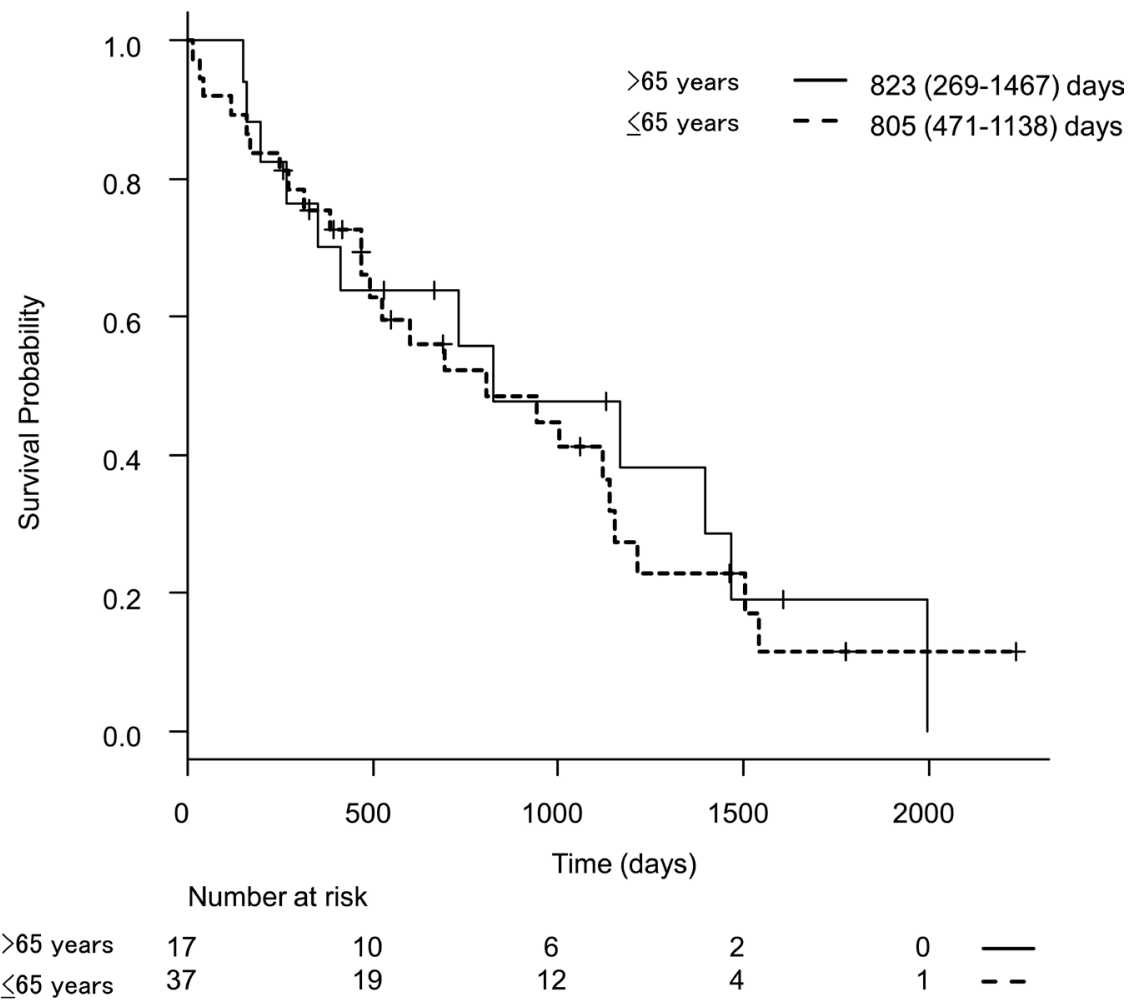

Figure 1. Overall survival (OS) in metastatic colon cancer patients receiving chemotherapy according to age.

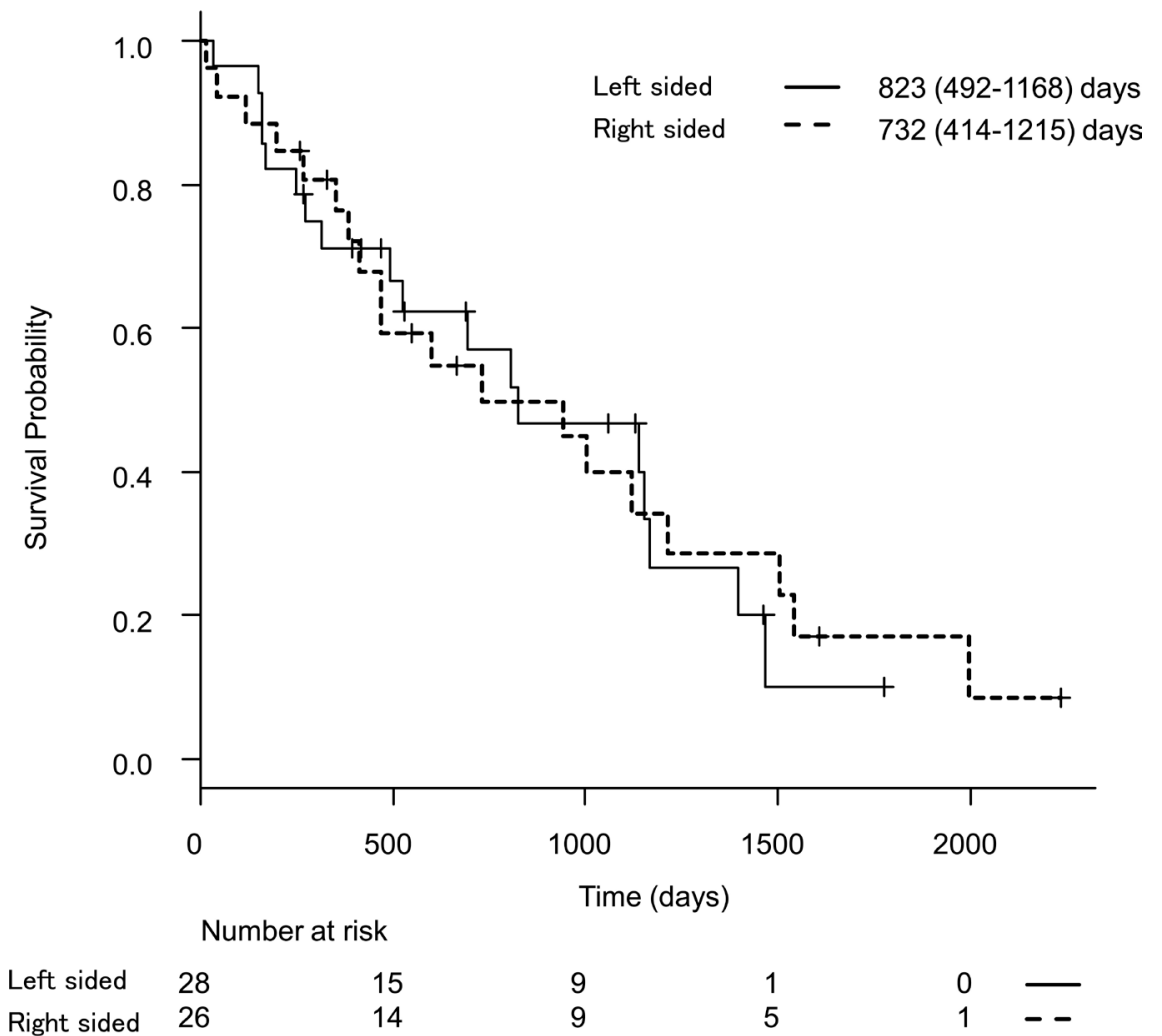

Figure 2. Overall survival (OS) in metastatic colon cancer patients receiving chemotherapy according to the primary lesion site. 


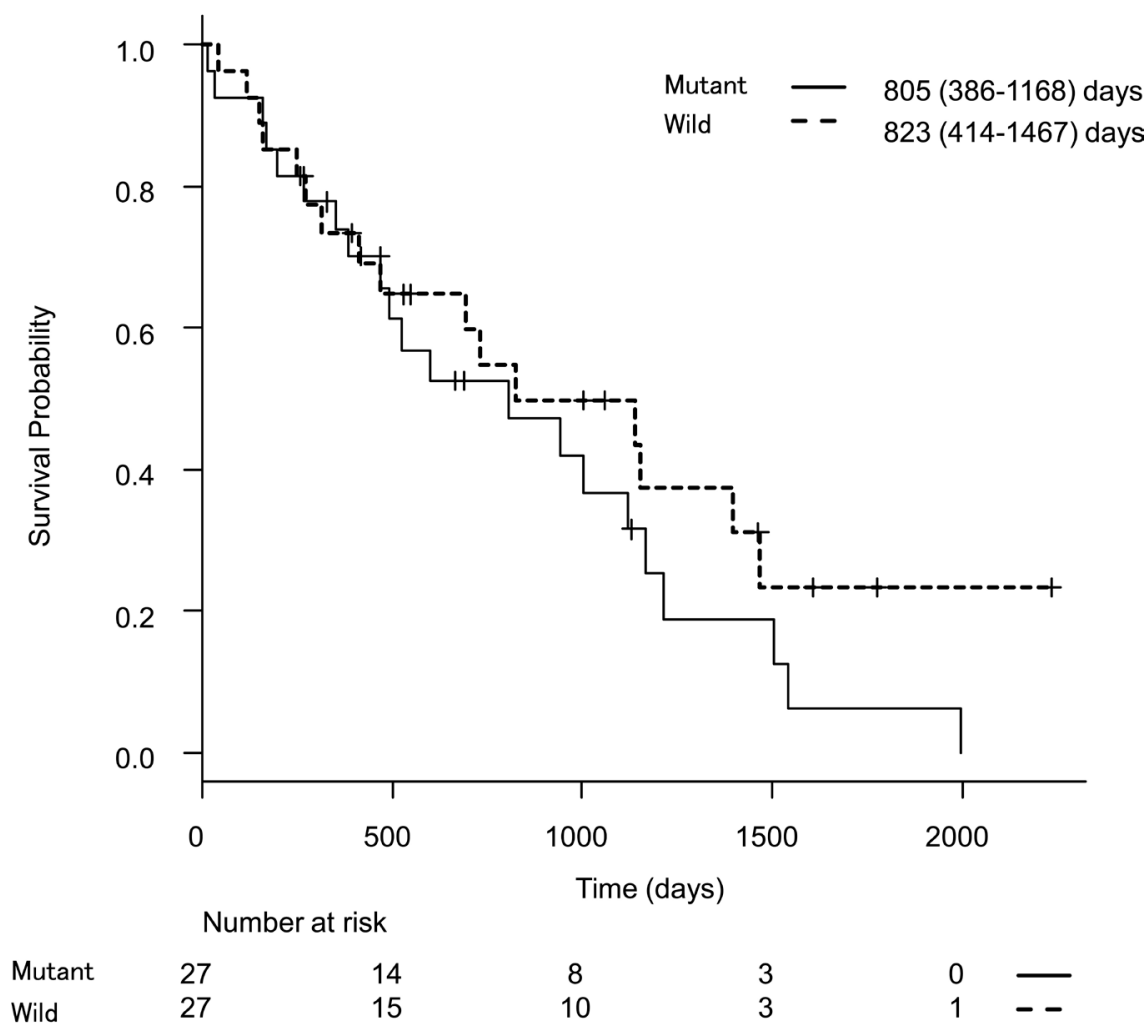

Figure 3. Overall survival (OS) in metastatic colon cancer patients receiving chemotherapy according to $R A S$ status.

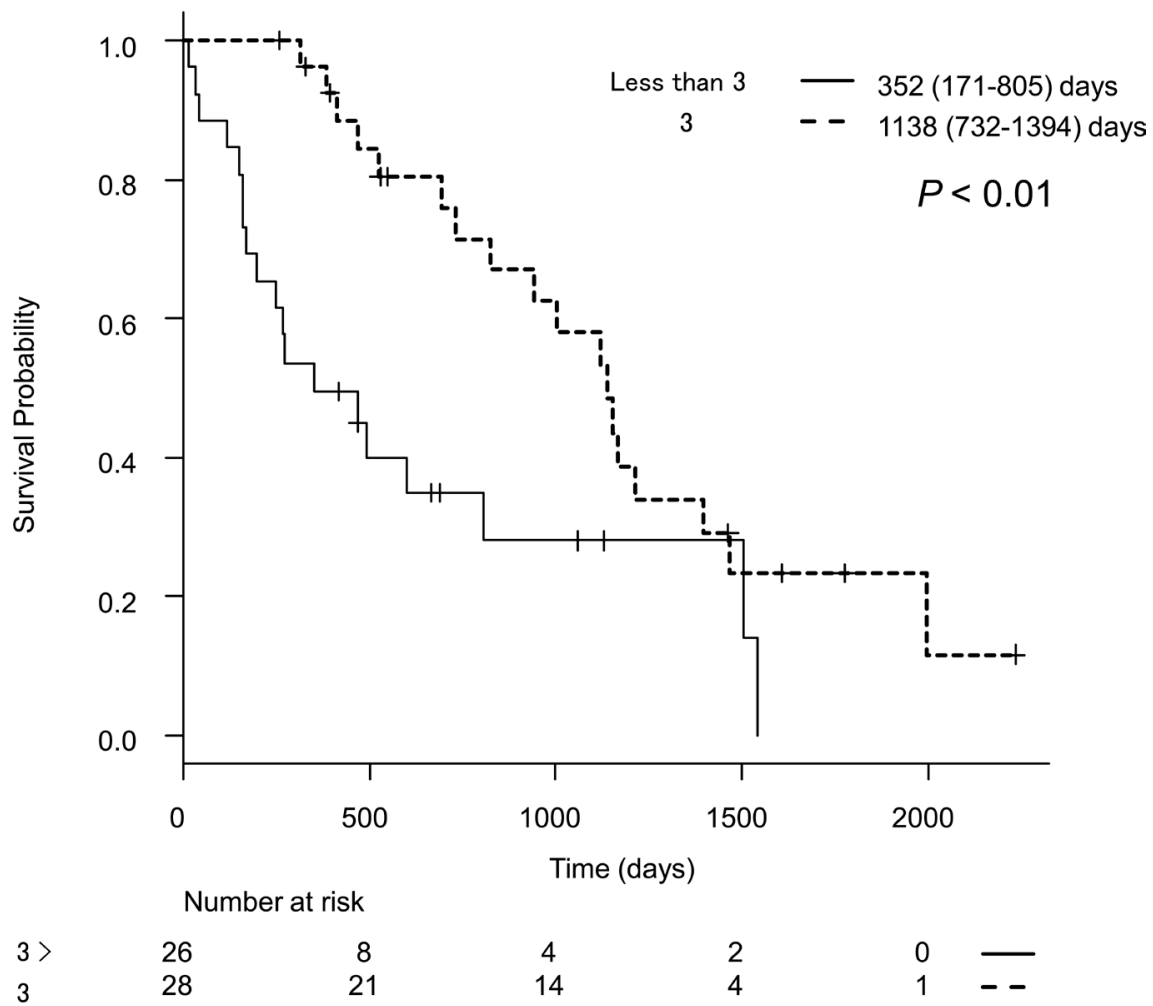

Figure 4. Overall survival (OS) in metastatic colon cancer patients receiving chemotherapy according to the number of cytotoxic agents administered. 


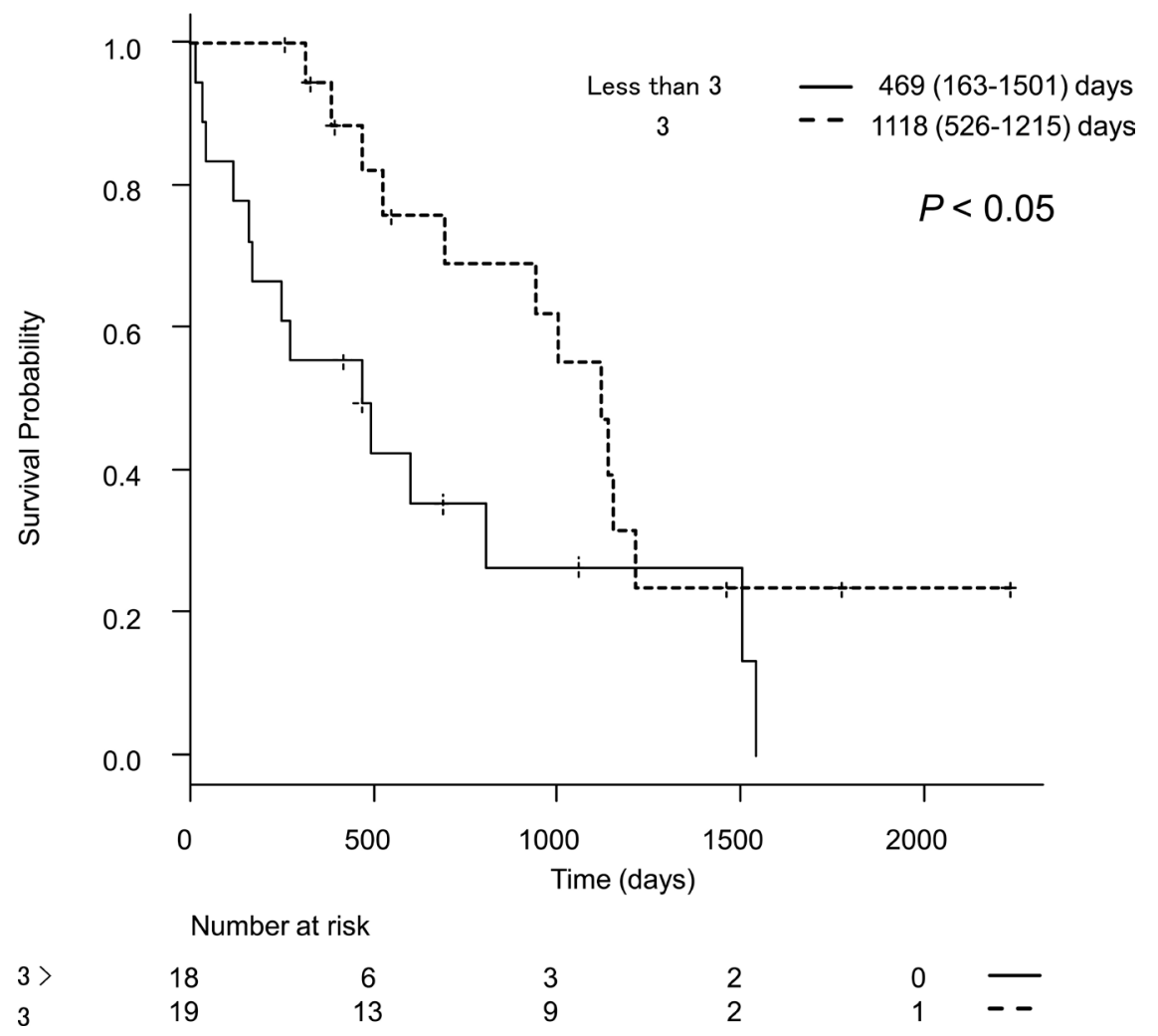

Figure 5. Overall survival (OS) in older patients with metastatic colon cancer receiving chemotherapy according to the number of cytotoxic agents administered.

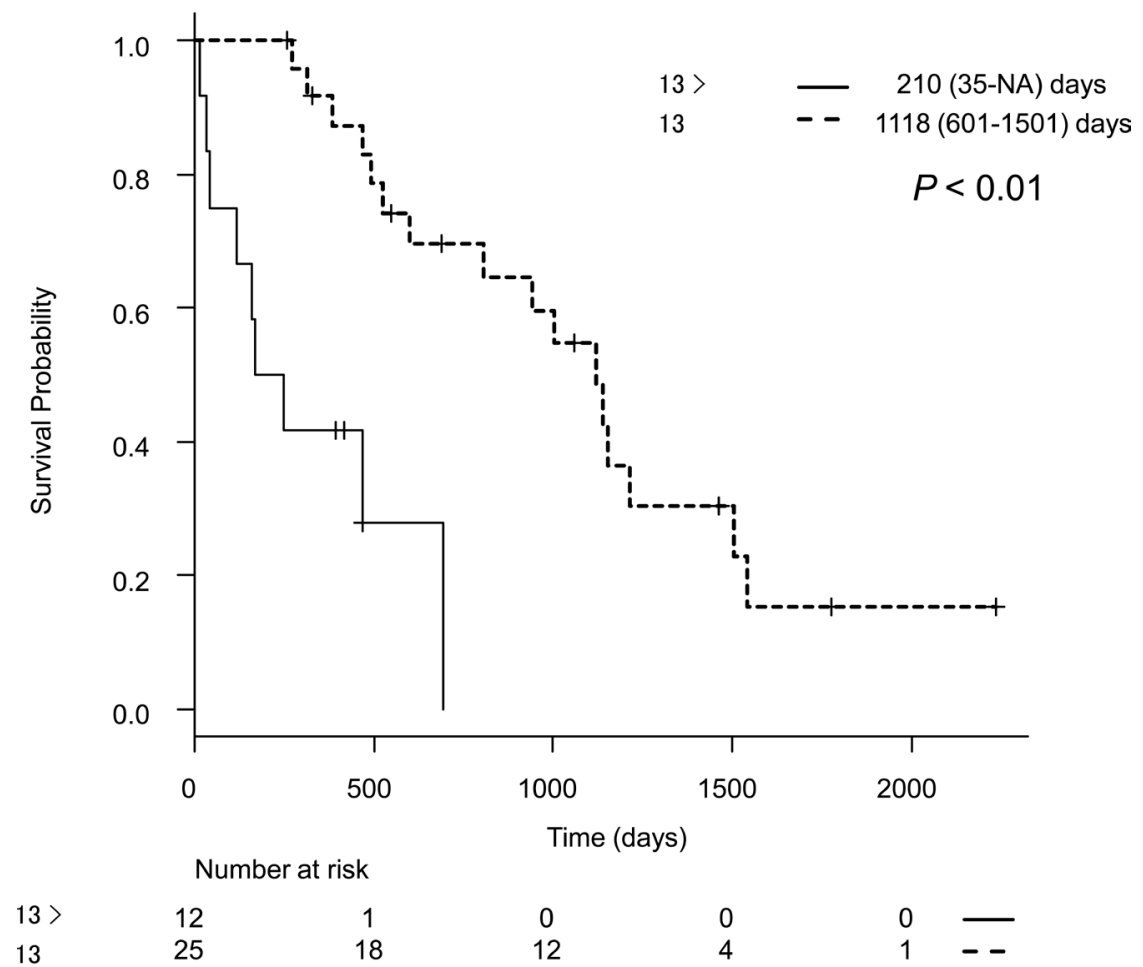

Figure 6. Overall survival (OS) in older patients with metastatic colon cancer receiving chemotherapy according to the Tokyo Metropolitan Institute of Gerontology score for competence. 
index of competence (hazard ratio 0.13 [95\% CI 0.05 - 0.38]; $P<0.001$, Table 1), but not as measured by IADL.

\section{Discussion}

In general, to optimize the survival outcomes of cancer patients, all patients should be exposed to active agents during the course of their disease [6]. The present analysis showed that metastatic colon cancer patients who received three cytotoxic agents survived longer than those administered fewer than three, which supported this notion. However, for older patients $\geq 65$ years of age, we found that the TMIG index score of competence was more relevant for survival than the number of cytotoxic agents used. Aging is associated with an accumulation of physiological deficits, which affects treatment tolerance and survival in elderly patients with cancer [18]. The evaluation of physical conditions at the initiation of chemotherapy is a significant predictor of treatment tolerance for elderly patients. A comprehensive geriatric assessment includes an assessment of the patient's ability to maintain independence in the community, such as taking transportation, shopping, managing money, doing housework, and communicating [21] [22]. These activities could be impaired due to comorbidity, cognitive

Table 1. Hazard ratios and 95\% confidence intervals (CI) of independent variables for overall survival of older patients with metastatic colon cancer $(n=37)$ (Cox proportional hazards regression).

\begin{tabular}{|c|c|c|c|}
\hline & Hazard ratio & $95 \% \mathrm{CI}$ & $P$ value \\
\hline \multicolumn{4}{|l|}{ Sex } \\
\hline Female & 1.00 & & \\
\hline Male & 1.44 & $0.61-3.45$ & 0.41 \\
\hline \multicolumn{4}{|l|}{ Site } \\
\hline Left-sided & 1.00 & & \\
\hline Right-sided & 1.16 & $0.47-2.86$ & 0.75 \\
\hline \multicolumn{4}{|l|}{$R A S$ status } \\
\hline Mutant & 1.00 & & \\
\hline Wild & 0.60 & $0.23-1.55$ & 0.29 \\
\hline \multicolumn{4}{|c|}{ Number of cytotoxic agents } \\
\hline $3>$ & 1.00 & & \\
\hline 3 & 0.89 & $0.30-2.60$ & 0.82 \\
\hline \multicolumn{4}{|l|}{ IADL } \\
\hline Others & 1.00 & & \\
\hline Full score & 0.27 & $0.02-3.40$ & 0.31 \\
\hline \multicolumn{4}{|l|}{ TMIG index } \\
\hline $13>$ & 1.00 & & \\
\hline Full score & 0.13 & $0.05-0.38$ & 0.000195 \\
\hline
\end{tabular}


disturbances, nutritional status, or psychological state. These skills can be measured by either an 8-item IADL scoring system or the TMIG index of competence, both of which are useful tools for identifying the vulnerability of geriatric patients. However, there are several differences between the two systems: the IADL system includes house affairs, which eventually differs in full scores between males (5 points) and females (8 points) [21], whereas the TMIG index of competence is superior to IADL in evaluating functional vulnerability beyond sex differences [22]. Although there are some limitations of our study due mainly to small sample size, performing a comprehensive geriatric assessment using the TMIG index before chemotherapy should be helpful in predicting the longevity of patients with metastatic colon cancer who were $\geq 65$ years of age.

\section{Conclusion}

The present analysis of elderly patients with metastatic colon cancer showed that a comprehensive geriatric assessment before chemotherapy was useful for predicting their prognosis.

\section{Conflicts of Interest}

The authors declare no conflicts of interest regarding the publication of this paper.

\section{References}

[1] Arnold, M., Sierra, M.S., Laversanne, M., Soerjomataram, I., Jemal, A. and Bray, F. (2017) Global Patterns and Trends in Colorectal Cancer Incidence and Mortality. Gut, 66, 683-691. https://doi.org/10.1136/gutjnl-2015-310912

[2] Yamazaki, K., Nagase, M., Tanigawa, H., et al. (2016) Randamized Phase III Study of Bevacizumab plus FOLFIRI and Bevacizumab plus mFOLFOX6 as First-Line Treatment for Patients with Metastatic Colorectal Cancer (WJOG4407G). Annals of Oncology, 27, 1539-1546. https://doi.org/10.1093/annonc/mdw206

[3] Van Custem, E., Lenz, H.J., Kohne, C.H., et al. (2015) Fluorouracil, Leucovorin, and Irinotecan plus Cetuximab Treatment and RAS Mutations in Colorectal Cancer. Journal of Clinical Oncology, 33, 692-700. https://doi.org/10.1200/JCO.2014.59.4812

[4] Douillard, J.Y., Siena, S., Cassidy, J., et al. (2014) Final Results from PRIME: A Randomized Phase III Study of Panitumumab with FOLFOX4 for First-Line Treatment of Metastatic Colorectal Cancer. Annals of Oncology, 25, 1346-1355. https://doi.org/10.1093/annonc/mdu141

[5] Loupakis, F., Cremolini, C., Masi, G., et al. (2014) Initial Therapy with FOLFOXIRI and Bevacizumab for Metastatic Colorectal Cancer. The New England Journal of Medicine, 371, 1609-1618. https://doi.org/10.1056/NEJMoa1403108

[6] Mahipal, A. and Grothey, A. (2016) Role of Biologics in First-Line Treatment of Colorectal Cancer. Journal of Oncology Practice, 12, 1219-1228. https://doi.org/10.1200/JOP.2016.018382

[7] Tournigand, C., Andre, T., Achille, E., et al. (2004) FOLFIRI Followed by FOLFOX6 or the Reverse Sequence in Advanced Colorectal Cancer: A Randomized GERCOR Study. Journal of Clinical Oncology, 22, 229-237. 
https://doi.org/10.1200/JCO.2004.05.113

[8] Chibaudel, B., Bonnetain, F., Tournigand, C., et al. (2011) Simplified Prognostic Model in Patients with Oxaliplatin-Based or Irinotecan-Based First-Line Cchemotherapy for Metastatic Colorectal Cancer: A GERCOR Study. Oncologist, 16, 1228-1238. https://doi.org/10.1634/theoncologist.2011-0039

[9] De Roock, W., De Vriendt, V., Normanno, N., Ciardiello, F. and Tejpar, S. (2011) $K R A S, B R A F, P I K 3 C A$, and PTEN Mutations: Implications for Targeted Therapies in Metastatic Colorectal Cancer. The Lancet Oncology, 12, 594-603. https://doi.org/10.1016/S1470-2045(10)70209-6

[10] The Cancer Genome Atlas Network (2012) Comprehensive Molecular Characterization of Human Colon and Rectal Cancer. Nature, 487, 330-337. https://doi.org/10.1038/nature11252

[11] Richman, S.D., Seymour, M.T., Chambers, P., et al. (2009) KRAS and BRAF Mutations in Advanced Colorectal Cancer Are Associated with Poor Prognosis but Do Not Preclude Benefit from Oxaliplatin or Irinotecan: Results from the MRC FOCUS Trial. Journal of Clinical Oncology, 27, 5931-5937.

https://doi.org/10.1200/JCO.2009.22.4295

https://www.researchgate.net/publication/38062622

[12] Venderbosch, S., Nagtegaal, I.D., Maughan, T.S., et al. (2014) Mismatch Repair Status and BRAF Mutation Status in Metastatic Colorectal Cancer Patients: A Pooled Analysis of the CAIRO, CAIRO2, COIN, and FOCUS Studies. Clinical Cancer Research, 20, 5322-5330. https://doi.org/10.1158/1078-0432.CCR-14-0332

[13] Andre, T., de Gramont, A., Vernerey, D., et al. (2015) Adjuvant Fluorouracil, Leucovorin, and Oxaliplatin in Stage II to III Colon Cancer: Updated 10-Year Survival and Outcomes According to BRAF Mutation and Mismatch Repair Status of the MOSAIC Study. Journal of Clinical Oncology, 33, 4176-4187.

https://doi.org/10.1200/JCO.2015.63.4238

[14] Weiss, J.M., Pfau, P.R., O’Connor, E.S., et al. (2011) Mortality by Stage for Rightversus Left-Sided Colon Cancer: Analysis of Surveillance, Epidemiology, and End Results-Medicare Data. Journal of Clinical Oncology, 29, 4401-4409. https://doi.org/10.1200/JCO.2011.36.4414

[15] Brule, S.Y., Jonker, D.J., Karapetis, C.S., et al. (2015) Location of Colon Cancer (Right-Sided Versus Left-Sided) as a Prognostic Factor and a Predictor of Benefit from Cetuximab in NCIC CO.17. European Journal of Cancer, 51, 1405-1414. https://doi.org/10.1016/j.ejca.2015.03.015

[16] Boeckx, N., Koukakis, R., Op de Beeck, L., et al. (2017) Primary Tumor Sidedness Has an Impact on Prognosis and Treatment Outcome in Metastatic Colorectal Cancer: Results from Two Randomized First-Line Panitumumab Studies. Annals of Oncology, 28, 1862-1868. https://doi.org/10.1093/annonc/mdx119

[17] Holch, J.W., Ricard, I., Stintzing, S., Modest, D.P. and Heinemann, V. (2017) The Relevance of Primary Tumor Location in Patients with Metastatic Colorectal Cancer: A Meta-Analysis of First-Line Clinical Trials. European Journal of Cancer, 70, 87-98. https://doi.org/10.1016/j.ejca.2016.10.007

[18] Hurria, A., Togawa, K., Mohile, S.G., et al. (2011) Predicting Chemotherapy Toxicity in Older Adults with Cancer: A Prospective Multicenter Study. Journal of Clinical Oncology, 29, 3457-3465. https://doi.org/10.1200/JCO.2011.34.7625

[19] Hall, P.S., Lord, S.R., Collinson, M, et al. (2017) A Randomised Phase II Trial and Feasibility Study of Palliative Chemotherapy in Frail or Elderly Patients with Advanced Gastroesophageal Cancer (321GO). British Journal of Cancer, 116, 472-478. 
https://doi.org/10.1038/bjc.2016.442

[20] Extermann, M. and Hurria, A. (2007) Comprehensive Geriatric Assessment for Older Patients with Cancer. Journal of Clinical Oncology, 25, 1824-1831. https://doi.org/10.1200/JCO.2007.10.6559

[21] Lawton, M.P. and Brody, E.M. (1969) Assessment of Older People: Self-Maintaining and Instrumental Activities of Daily Living. The Gerontologist, 9, 179-186. https://doi.org/10.1093/geront/9.3_Part_1.179

[22] Koyano, W., Hashimoto, M., Fukawa, T., Shibata, H. and Gunji, A. (1993) Functional Capacity of the Elderly: Measurement by the TMIG Index of Competitive. Japanese Journal of Public Health, 40, 468-474.

[23] Therasse, P., Arbuck, S.G., Eisenhauer, E.A., et al. (2000) New Guidelines to Evaluate the Response to Treatment in Solid Tumors. Journal of the National Cancer Institute, 92, 205-216. https://doi.org/10.1093/jnci/92.3.205

[24] Kanda, Y. (2013) Investigation of the Freely-Available Easy-to-Use Software "EZR" (Easy R) for Medical Statistics. Bone Marrow Transplantation, 48, 452-458. https://doi.org/10.1038/bmt.2012.244 